\title{
1-507-3
}

\section{Contribution to Development of Remedies for COVID-19: Focusing on Eritoran}

\section{Kappei Tsukahara}

Eisai Co., Ltd.

Eritoran (E5564) is Eisai's in-house discovered and developed investigational TLR4 (Toll-Like Receptor 4) antagonist created with natural product organic synthesis technology. It is a structural analogue of Lipid A, which is an activator of endotoxins of bacteria. It has been previously observed to be safe in 14 clinical studies including a large Phase 3 randomized trial in severe sepsis. We are participating in the international network REMAP-CAP-COVID (Randomized, Embedded, Multi-factorial, Adaptive Platform-Community Acquired Pneumonia COVID) which aims for novel coronavirus medicine development through drug repurposing, and began an international collaborative clinical trial in October 2020 which is designated for confirmed novel coronavirus patients who are hospitalized and are in a progressing disease state. It is hoped that through suppressing the most upstream TLR4 activity which controls production of multiple cytokines, the cytokine storm in patients can be suppressed and pneumonia can thus be prevented from becoming severe. 